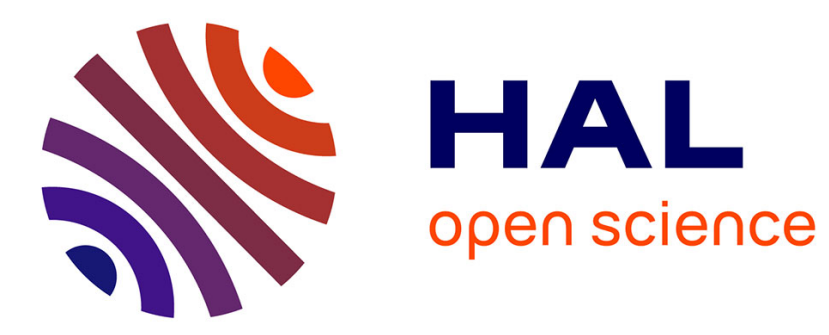

\title{
Building Trust Between Users and Telecommunications Data Driven Virtual Assistants
}

\author{
Marta Perez Garcia, Sarita Saffon Lopez
}

\section{To cite this version:}

Marta Perez Garcia, Sarita Saffon Lopez. Building Trust Between Users and Telecommunications Data Driven Virtual Assistants. 14th IFIP International Conference on Artificial Intelligence Applications and Innovations (AIAI), May 2018, Rhodes, Greece. pp.628-637, 10.1007/978-3-319-92007-8_53 . hal-01821083

\section{HAL Id: hal-01821083 \\ https://hal.inria.fr/hal-01821083}

Submitted on 22 Jun 2018

HAL is a multi-disciplinary open access archive for the deposit and dissemination of scientific research documents, whether they are published or not. The documents may come from teaching and research institutions in France or abroad, or from public or private research centers.
L'archive ouverte pluridisciplinaire HAL, est destinée au dépôt et à la diffusion de documents scientifiques de niveau recherche, publiés ou non, émanant des établissements d'enseignement et de recherche français ou étrangers, des laboratoires publics ou privés. 


\title{
Building Trust Between Users and Telecommunications Data Driven Virtual Assistants
}

\author{
Dr. Marta Perez Garcia ${ }^{1[0000-0002-4109-1653]}(\square)$ and Sarita Saffon Lopez ${ }^{2}$ \\ ${ }^{1}$ Telefonica R\&D, Madrid, Spain \\ 2 Telefonica R\&D, Madrid, Spain \\ marta.perezgarcia@telefonica.com \\ sarita.saffonlopez@telefonica.com
}

\begin{abstract}
When we think about traditional technology services, such as the Internet and popular smartphone apps, interaction or experience related words tend to come to our mind. However, when we shift to more disruptive and breakthrough technologies, such as big data, IoT or Artificial Intelligence (AI), trust and security become a key challenge as the environments in which the personal information is stored and used to extract value changes in ways that are difficult to understand for normal users. This study explores the current challenges to trust and rely on a Virtual Assistant and establishes a series of constructs to build trust in a Telecoms Data Driven Virtual Assistant by considering aspects from an embryonic idea of the AI to its final articulation as a digital service. It examines a series of multi-methodology research interactions to help shape and refine the service, not only to build trust in the AI but also in the organisation behind it.
\end{abstract}

Keywords: Artificial Intelligence, Data Driven Virtual Assistants, Trust, $\mathrm{Hu}-$ man Computer Interaction, Telecommunications

\section{Introduction}

Since ancient times, humans have had the curiosity to explore their own cognitive capacities, also referred to as intelligence, and how to replicate them in their own inanimate creations [1]. Literature, myths and history itself prove this; from Von Kempelen's “Turk” automaton playing chess with Napoleon, to Mary Shelly's Frankenstein, to Alan Turing's code deciphering machine, until the development of Data Driven Virtual Assistants (DDVA) [2] [3]. The relationship between the human mind and machines has grown so strong that the language used to refer to both is almost analogous, with terms such as memory, hard drive, sensors, neurons, and so on. This discipline then, of studying the human cognitive competences and trying to replicate them, has become a science called Artificial Intelligence (AI). From competing in games such as chess [4]; to being part of strategic war weapons [5], to being able to recognize speech [6], and now to current uses in industries such as logistics, retail, education and financial services [7]. Furthermore, the future uses of AI are expected to apply to diverse areas, such as medicine, where surgeries might be done by automatons [8]. After briefly reviewing AI's past, present and future, it is clear that it has become part of our lives, even though humans continue to doubt, and even fear the power that 
AI may reach. This assertion is supported by Pega Systems global 6.000 consumer study [9] where $70 \%$ of the participants reported that they feared AI and $24 \%$ are worried about robots taking over the world.

\section{$2 \quad$ What does trust mean and why is it important?}

Trust is defined as someone's willingness to become susceptible regarding a certain issue, context or information [10]. This is the reason why trust is of special importance when it comes to human-computer interaction, where users need to trust and rely on the digital agents or virtual assistants because they have to act on their behalf [11]. In the context of online versus offline, Corritore et al [10] identified three core aspects that need to be built in order to generate trust: ease of use, risk involved and technology credibility. Sas and Khairuddin [12] go beyond this, establishing there are two different types of trust when it comes to people interacting with technology: (1) trust between users and technology itself; and (2) trust between users who interact with technology. In the case of Virtual Assistants, trust is more focused on the first one, trust between users and technology, as it is a one-to-one relationship and there is no communication or information sharing with other party. In fact, when it comes to this information sharing between the user and the AI technology, it becomes obvious that not all of the information is of the same quality and usefulness, so allowing a system to dissect it is the first step towards trust [13]. Ultimately, the objective would be to enable these artificial intelligence virtual assistants to take care of issues with no intervention or supervision of the user [14]. Many articles have been written arising this trust issue between users and artificial intelligence but there is however little work done on the investigation of their key constructs needed to generate trust between these parties.

\section{$3 \quad$ Key challenges in trusting an AI}

Northstar Research Partners [15] carried out a survey study that explored the awareness and acceptance of AI from a consumer perspective. It highlights three concerns that participants reported around the subject of AI, which constitute the focus of building and maintaining trust.

\subsection{A lack of knowledge of AI does not help build reliance}

By definition, there are two types of AI: weak and strong [16]. On one hand, only weak AI exists currently, which is based on programmed response-powered algorithms. Therefore, this is a supervised process that does not comprehend the commands it receives, but classifies them according to a pre-set data base of responses, and only simulates a human-like experience. On the other hand, strong AI, not yet created but in the process, has a mind of its own, hence it creates clusters and associations in order to provide a response. However, do users know this? Are they aware of how artificial intelligence works? Pega Systems study [9] reports that more than 50\% of its 6,000 - 
customer base are not aware of ever interacting with AI technology. This fact highlights the massive gap between people's perceptions, awareness and conscience around AI. Most information about AI users have is what they have seen in futuristic science fiction movies where machines take over the world, so it becomes crucial to create support, coordination, explanation groups and initiatives [11] to make people knowledgeable of the benefits and potential of AI to enrich their lives. As MIT professors Erik Brynjolfsson and Andrew McAfee [17] explain, AI is the base of what they refer to as the second machine age, resembling a new industrial revolution, where "computers and other digital advances are doing for mental power... what the steam engine and its descendants did for muscle power." (pp. 7-8). The process towards trusting this technology will require exposure and experience, such as it did in the $18^{\text {th }}$ century with the Industrial Revolution. In summary, people mistrusted machines at the beginning of the Industrial Revolution like people mistrust AI today because, as the Pega Study [9] shows, they do not even know what AI actually is.

\subsection{Mistrusting AI to solve complex problems}

Every customer is different and as such, their problems have very diverse contingencies. How can a DDVA be smart enough to understand all the details, specifics of thousands and millions of customers? Research shows that existing AI relies on human monitoring, a best practice referred to as Human-in-the-Loop [18], which is based on generating machine learning models that, in case of uncertainty or in contexts with a low level of confidence, yields the decision making to a human mind. This feedback is used to learn and improve algorithms over time. AI is not currently taking over the complex functions but the easy ones, while real people still work on the hard functions. This case is illustrated in current automatic pilot commercial planes like the Boing 777 [19] and self-driving cars as the Tesla autopilot mode in their models X, S and 3 [20], where a human is always in the driving seat to take control if needed. When the AI systems need autonomy to perform tasks and make decisions on behalf of users, it becomes essential to develop trust on the AI actions in order to use them and adopt them as assistants in people's daily lives [21]. Currently, a human is always there controlling cases of doubt or ambiguity, for no machine is perfect, as no human is [22], so there is no need to separate AI from humans, since various trials such as with Centaur Chess [23] and IBM Watson [24] proved that human minds and machines work better together than separately [25].

\subsection{Managing and handling personal and private data}

Other concern that increases distrust on $\mathrm{AI}$ is the uncomfortable necessity of sharing our personal data with an AI system, and not being sure of the ethical guidelines that it follows [26]. For instance, to assure ethical guidelines, it is important AI developers address the three AI factors suggested in The Cambridge Handbook of Artificial Intelligence [27]: (1) being transparent to inspection, (2) being predictable and (3) being non-operable, with the objective of becoming trust-generating features. This could be done by explaining the algorithms behind the system to support the transparency and 
predictability of the system [28]. Banavar [29] also proposes various tasks in order to achieve trust and reassurance. For example, before launching an AI system, he suggests to run field testing for ethics-related design issues, and continue to elicit user feedback throughout the deployment to monitor other potential ethical problems. When considering a product or service for a DDVA, ethics programs should be embedded in the AI system so they can reduce the risks and guide the system into values and principles in which it has to base its own behaviour [29]. These guidelines require research and interdisciplinary teams of professionals in ethics and in AI development, to find a common language that will ensure the proper and practical implementation of ethical program in the machines [30]. For ensuring that these best practices are respected and enforced, current players in the AI field (Amazon, Google, Facebook, IBM, etc.) have already formed an alliance, in which they pursue various ethical topics, and develop new ones to safeguard the transparency and safety of data used by AIs [31].

\section{Best practices in AI: What to do and what to avoid}

Trust building in any part of people's lives is affected by first impressions [32]. With DDVA, it is no different. According to psychologist Albert Mehrabian [33], 38\% of first impressions depend on the tone of voice, and only $7 \%$ on the words used. This includes using silences between sentences so that the person listening has time to process the information given, and having fillers just like "mmm" or "well", all to create the feeling of talking to a human being and therefore developing trust [34]. A second aspect that has to be considered is the gender of the DDVA, some key players in this area, such as Apple, Google or Amazon, have ensured that the device not only has a human-like tone of voice but a female one, as they are perceived as warmer [35], more trustworthy [36], easier to understand [37], in summary, easier to like. However, the human like aspect of the DDVAs cannot go to an extreme. Dr. Masahiro Mori [38] developed in 1970 the theory of the Uncanny Valley, which states that as the AI becomes more human-like there is an increase in the levels of acceptability and affinity with the device, but if it resembles too much human, there is a dramatic decrease of approval reaching even negative likeness and unsettling feelings [38]. This is the reason why many organisations are now going into the opposite direction, trying to avoid this Uncanny Valley. For instance, newly competitors have shifted from previous DDVA trend of naming their devices with human-like names as Alexa or Cortana, to more neutral and unbiased names like Bixby by Samsung and Tmall by Alibaba. Even though these best practices are being published, implemented and protected by the DeepMind Alliance, a well-established AI research organisation, there exist various cases of AI failures from where other learnings can rise. An example is Microsoft's Tay, an AI chatbot which used people's Twitter conversations to generate her own, and was tricked by users to learn and produce racist and other harmful tweets. Additionally, Tay was modelled as a teenager girl, pushing the feminisation of the AI, worsening the situation [39]. Another example of a not so successful outcome is Facebook's AI robots, Alice and Bob, which were disconnected because, while performing a negotiation between them, they generated a language that was incomprehensible for human beings [40]. 
These cases represent examples that feed fears inherent in the human mind, and inhibits them from trusting AI systems.

\section{The Context}

This research study takes place within a multinational telecoms organisation because it reflects rather clearly two challenges the literature review has highlighted around trusting AI: (1) AI taking over complex points of interaction between users and humans where problem solving is essential; and (2) the aversion towards an AI handling personal data (consumption of services, behaviours, habits, etc.). A Telecoms DDVA could work as an artefact to save time and effort, helping users make the most of their hired services and improve overall customer experience satisfaction [41]. The principles that appear in the findings section of this paper are being applied to Telefonica (trading as Movistar, Vivo and $\mathrm{O} 2$ in more than twenty countries) and its cognitive intelligence Aura. However, the study has been carried out as an exploratory study and has been conducted in general terms, evaluating the results as a category (not associating it to a specific brand) and recruiting participants from all the major Telecoms providers in Spain.

\section{$6 \quad$ Methodology and Sample}

To explore and tackle the issues highlighted in the literature, two ad-hoc user-centred research activities in Spain were conducted:

1. Sixteen in-depth interviews to find out perceptions around the concept, the value proposal and use cases. This was tested with a sample of six current DDVA users, six ex users and four aware but not current users. Two people of three age ranges (25-35, $36-45,46-55)$ formed each of the groups.

2. Five hundred online questionnaires to explore the personality of current DDVAs and future interests, of which two hundred and nine participants were current users of DDVAs, a hundred and one ex users, and hundred and one aware but not current users. In each of these groups there was a mix of ages: 16-24, 25-34, 35-44 and 45-54 years old.

All participants were of medium- high socio economic level, living in capital cities and in proportion of $50 \%$ men and $50 \%$ women. The reason behind all these recruitment parameters was to understand habits, expectations, fears and perceptions of different segments with different approaches and usage of technology.

\section{$7 \quad$ Findings}

These different research activities that took place during this exploratory study helped identify a series of key aspects in the development of trust with a telecoms DDVA: 


\subsection{How should a Telecoms Data Driven Virtual Assistant be?}

After identifying a suitable name for the DDVA, this study sought to understand the personal qualities it should have in order to emotionally connect with users. In the quantitative phase of this study, the 500 questionnaires, users were presented with a series of characters, skills and personality aspects to determine current and future perception of VAs. This helped understand the relationship between functional and emotional benefits users experiment with this type of Artificial Intelligence. In terms of characters, users were given a wide range of options to articulate the way they would perceive a Telco DDVA. Among these options, $60 \%$ of current 299 VA users agreed that they would interact with a robot-like character, contrasted by the $0 \%$ and $11 \%$ that chose mother or friend, respectively. This remote expectation of a personified human-like DDVA and instead an expectation of a pure technological, digital and artificial machines, confirms once more the Uncanny Valley theory of not humanising AI in excess [38]. In terms of personality traits, users expect DDVAs to be precise, especially when serving them information and answers. A suggested future improvement of DDVA personality is creativity, which is something they currently lack. Users also desire a Telecoms DDVA to be proactive, able to make intelligent suggestions and teach them how to make the most out of their services. However, users also expressed that they still lack the trust in AI to handover decision making, so we believe the proactivity of DDVAs must have a clearly defined scope. In summary, the personality traits (precision, creativity, proactivity) users expect and desire from a Telecoms DDVA have the potential of fostering positive connotations that could influence the perception of the Telecommunication Organization itself.

\subsection{Transferring human roles to $\mathrm{AI}$}

The in-depth individual interviews explored the different aspects regarding the concept and value proposal of the DDVA. Within this context, users highlighted the fact that one of the DDVA objectives should be to improve customer experience and customer care with a Telecoms provider. This led to two major findings: (i) Users considered this would represent a massive change for customer care experience and many of them felt, a priori, reluctant to change. However, after a first moment of doubt, most users started balancing pros and cons of current situation and the proposed one and considered the optimisation of time and resources that a DDVA would have for them resulted on a more positive perceived customer care relationship; (ii) Secondly, users also would like to have a backup person to talk to when the DDVA is not able to solve their problem, following the Human In-The-Loop practice [18]. These findings point out to the fact that people do not consider the DDVA will satisfy all their requests, and consider that they would not remain unsolved when combining human and AI forces [25]. This led to a higher level of satisfaction of the DDVA concept proposed, while establishing a potential scenario to merge both worlds in order to enhance customer service and strengthen the value proposition. 


\subsection{The importance of the decision-making process}

In this case, users were given a series of stimulus data in the form of different use cases the DDVA could carry out in order to improve their relationship with the Telecoms provider. This led to a discussion around the articulation of these scenarios, exploring the role the DDVA would play. The results of this study showed users are very interested in a DDVA that is proactive, makes suggestions and has a didactic function for users in order to learn the possibilities it offers. They showed an interest in receiving alerts about anomalies in their hired telco services. However, a key insight that was identified was the importance of the decision-making process in this type of scenarios. Users implied that even when a DDVA could potentially solve a problem with their services, they expect the DDVA to alert them and ask for permission to start sorting out the incidence. This suggests users are at a very early stage within the trust path towards independent DDVAs and still want to ensure they have the power and authority to control them. They are not interested yet in a DDVA that is able to make independent decisions on their behalf, even if this is for their own good. This takes the results back to perception of the DDVA as a robot or servant exposed previously.

\subsection{Gathering and handling personal data}

The literature has highlighted how personal data handling is one of the main challenges when trusting AI [15]. This was a core aspect in this study as the Virtual Assistant is driven by the data gathered in the Telecoms organisation and the data that the user keeps generating when making use of its telecoms services. The interviews with users have helped identify two moments of truth around this issue: (1) The first one happens when a person realises the quantity of data the Telecoms has about its customers, which generates a sense of surprise and agitation, however, after this brief moment, users tend to have (2) the second moment of truth, by which they understand that information is already within their service provider, it is acceptable they have it. This thought calms them down as they realise nothing bad has ever happened to them around data handling with their telecoms provider.

\section{Conclusions}

Building trust is a complex task, as it rests on a combination of several characteristics or features. The different research activities have helped identify a series of aspects that are essential to the start building trust between a user and a Telecoms DDVA, so the learnings from the different experiments are currently being applied to Aura, Telefonica's Data Driven Virtual Assistant. Firstly, when building a DDVA, a series of characteristics to shape its personality are required. This study has pointed out the importance of being precise in the information offered and the level of quality in the results the DDVA shows. In the future, a more creative personality could be built on top of efficiency. These personality qualities, are currently being applied to Aura, so the team is working on the efficiency and precision with which Aura can answer a series 
of given questions to users. Furthermore, deepening the knowledge around these attributes is key to build the desired personality and that works with the mother brand. A second issue that has emerged is the articulation of the decision-making process, which the qualitative interviews have shown is placed on the user. Even when dealing with a contingency or problem, users are not yet prepared for the DDVA to make decisions on its own. This study highlighted the importance that having control has for the user so Aura will notify users of any abnormality so that the user is the one with the power, the control over his own telecoms services and it is the one who makes the decisions. This is something that could evolve in the future, as these type of products and services become increasingly integrated in everyday life, but not yet at this early stage. Consequently, when this type of DDVA adoption evolves, Aura's team would like to test if users trust her enough to enable her to make decisions on her own (benefiting the user). In terms of the implications of the DDVA taking over certain interactions in the customer experience, specially its potential implementation in customer care, users have shown an interest toward the time and effort saving the DDVA could bring them in this type of situation, improving their level of satisfaction with the telecoms organisation. The trade-off they require is to be redirected to a person when the DDVA is not able to solve the issue they are trying to sort out. As for privacy and security of personal data, this study has suggested that the brand equity of the organisation providing the DDVA plays a key role in addressing this challenge. An organisation that is not able to build trust and confidence in its customers will probably not be able to generate trust in a DDVA, no matter the value proposition.

\section{References}

1. Villena, J., Crespo, R. and García, J.: Inteligencia en Redes de Comunicaciones 01 Historia de la Inteligencia Artificial. Universidad Carlos III de Madrid, Madrid (2012).

2. McCorduck, P., Minsky, M., Selfridge, O. and Herbert, S.: History of Artificial Intelligence. IJCAI, pp. 951-954. AAAI Press, Cambridge (1977).

3. Bolter, D.: Turing's Man: Western culture in the Computer Age. UNC Press Books, Chapel Hill (2014).

4. DeCoste, D.: The Future of Chess-Playing Technologies and the Significance of Kasparov Versus deep Blue. In: Proceedings of the 4th AAAI Conference on Deep Blue Versus Kasparov: The Significance for Artificial Intelligence, pp. 9-13. AAAI Press (1997)

5. Teuscher. C.: Alan Turing: Life and Legacy of a Great Thinker. $1^{\text {st }}$ edn. Springer Science + Business Media, Germany (2004).

6. Kuligowska, K., and Lasek, M..: Virtual assistants support customer relations and business processes. In: 10th International Conference on Information Management, pp. 155-164. Gdańsk (2011).

7. Bahrammirzaee, A.: A comparative survey of artificial intelligence applications in finance: artificial neural networks, expert system and hybrid intelligent systems. Neural Computing and Applications 19(8), 1165-1195 (2010). 
8. Grace, K., Salvatier, J., Dafoe, A. Zhang, B. and Evans, O.: When Will AI Exceed Human Performance? Evidence from AI Experts. ArXiv e-prints (2017).

9. Pega Systems. What consumers really think about AI: A Global Study, https://www.pega.com/ai-survey, last accessed 2018/01/18.

10. Corritore, C., Kracher, B. and Wiedenbeck, S.: On-line trust: concepts, evolving themes, a model. International Journal of Human Computer Studies 58 (6), 738758 (2003).

11. Castelfranchi, C.: The role of trust and deception in virtual societies. International Journal of Electronic Commerce 6(3), 55-70 (2002).

12. Sas, C., and Khairuddin, I.: Design for Trust: An exploration of the challenges and opportunities of bitcoin users. In: Proceedings of the 2017 CHI Conference on Human Factors in Computing Systems, pp. 6499-6510. ACM, New York (2017).

13. Marsh, S.: Trust and Reliance in Multi-Agent Systems: A Preliminary Report. In: MAAMAW'92, 4th European Workshop on Modelling Autonomous Agents in a Multi-Agent World, Rome (1992).

14. Castelfranchi C., and Falcone R.: Social trust: A cognitive approach. In C. Castelfranchi and Y.H. Tan (eds.), Deception, Fraud and Trust in Virtual Societies. Kluwer, Dordrecht (2001).

15. Northstar Research Partners. AI today, AI tomorrow: Awareness, acceptance and anticipation of AI: A global consumer perspective, http://pages.arm.com/rs/312SAX-488/images/arm-ai-survey-report.pdf, last accessed 2018/01/21.

16. Kerns, J: What's the Difference Between Weak and Strong AI. Machine Design, http://www.machinedesign.com/robotics/what-s-difference-between-weak-andstrong-ai, last accessed 2018/01/21.

17. Erik Brynjolfsson, E., and McAfee, A.: The second machine age: Work, progress, and prosperity in a time of brilliant technologies. WW Norton \& Company, New York (2014).

18. Dautenhahn, K.: The art of designing socially intelligent agents: Science, fiction, and the human in the loop. Applied artificial intelligence 12(7-8), 573-617 (1998).

19. Sha, S., Bumataria, R., Choudhary, A. and G. Bandhnia, G: Primary Flight Control of Boeing-777. International Journal of Advanced Research in Engineering, Science \& Technology (IJAREST), 2 (5) (2015)

20. Tesla: Full Self-Driving Hardware on All Cars, https://www.tesla.com/autopilot, last accessed 2017/09/02

21. Glass, A., McGuinness, D. and Wolverton, M.: Toward establishing trust in adaptive agents. In: Proceedings of the 13th international conference on Intelligent user interfaces, pp. 227-236. ACM, New York (2008).

22. Negnevitsky, M.: Artificial Intelligence: A Guide to Intelligent Systems. $2^{\text {nd }}$ edn. Pearson Education Limited, Essex (2005).

23. Scharre. P.: Centaur Warfighting: The False Choice of Humans vs. Automation. Temple International \& Comparative Law Journal 30 (1), 151 (2016).

24. Rachlin, H.: Making IBM's computer, Watson, human. The Behaviour Analyst 35(1), 1-16 (2012).

25. Bunch, L., Bradshaw, J., Hoffman, R. and Johnson, M.: Principles for humancentered interaction design, part 2: Can humans and machines think together?. IEEE Intelligent Systems 30(3), 68-75 (2015). 
26. Abascal, J. and Colette, N.: Moving towards inclusive design guidelines for socially and ethically aware HCI. Interacting with computers $17(5), 484-505$ (2005).

27. Frankish, K., and Ramsey, W. eds.: The Cambridge handbook of artificial intelligence. Cambridge University Press, Cambridge (2014).

28. Gibb, J.: T-Group theory and laboratory method: Innovation in re-education Climate for trust formation. John Wiley and Sons, New York (1964).

29. Banavar. G.: Learning to trust artificial intelligence systems. IBM Research, https://www.research.ibm.com/software/IBMResearch/multimedia/AIEthics Whitepaper.pdf, last accessed 2017/08/23.

30. Anderson, M. and Leigh Anderson, S.: Machine ethics. Cambridge University Press, Cambridge (2011).

31. Industry Leaders Establish Partnership on AI Best Practices. IBM News, https://www-03.ibm.com/press/us/en/pressrelease/50668.wss, last accessed 2017/08/29

32. Yu, M., Saleem, M. and Gonzalez, C.: Developing trust: First impressions and experience. Journal of Economic Psychology 43, 16-29 (2014).

33. Mehrabian. A.: Silent messages: Implicit communication of emotions and attitudes. $2^{\text {nd }}$ edn. Wadsworth, Belmont (1981).

34. Kedem. A.: Is Siri lying to you? Knowing when a bot sounds trustworthy is the next step in digital security, https://q.com/928201/is-siri-lying-to-you-knowingwhen-a-bot-sounds-trustworthy-is-the-next-step-in-digital-security/, last accessed 2018/01/18.

35. Mitchell, W., Ho, C., Patel, H. and MacDorman. K.: Does social desirability bias favour humans? Explicit-implicit evaluations of synthesized speech support a new HCI model of impression management. Computers in Human Behaviour 27(1), 402-412 (2011).

36. Siegel, M., Breazeal, C., and Norton, M.: Persuasive robotics: The influence of robot gender on human behaviour. IROS 2009. IEEE/RSJ International Conference on Intelligent Robots and Systems, pp. 2563-2568. IEEE (2009).

37. Sokhi, D., Hunter, M., Wilkinson, I. and Woodruff, P.: Male and female voices activate distinct regions in the male brain. Neuroimage Journal 27(3), 572-578 (2005).

38. Mori, M.: The Uncanny Valley. Energy 7(4), 33-35 (1970).

39. Neff, G. and Nagny. P.: Automation, Algorithms, and Politics| Talking to Bots: Symbiotic Agency and the Case of Tay. International Journal of Communication 10, 17 (2016).

40. Bradley, T.: Facebook AI Creates Its Own Language In Creepy Preview Of Our Potential Future, https://www.forbes.com/sites/tonybradley/2017/07/31/facebookai-creates-its-own-language-in-creepy-preview-of-our-potential-future/\#473aba81292c, last accessed 2018/01/18.

41. Perez, M.: The potential of Data-Driven Virtual Assistants to enhance Customer Experience in the Telecommunications Industry. In: $9^{\text {th }}$ Interaction South America Conference., Floripa, (2017). 